\title{
An Integrated ISFETs Instrumentation System in Standard CMOS Technology
}

\author{
Wai Pan Chan, Bhusana Premanode, and Christofer Toumazou, Fellow, IEEE
}

\begin{abstract}
This paper describes an integrated ISFETs instrumentation system in a $0.18 \mu \mathrm{m}$ 1-poly-6-metal CMOS process. The chip is able to compute the average of CMOS ISFETs' threshold voltages by using an averaging array employing global negative current feedback. In addition, neither reference voltage nor current is required to set up the sigma-delta modulator because the internal signal is converted and processed in the frequency domain. The chip operates at $3.3 \mathrm{~V}$ for the analog blocks and the digital input/output blocks, and at $1.8 \mathrm{~V}$ for the core digital logic. It achieves 8 bits accuracy under $80 \mu \mathrm{W}$ static power consumption. The die area is $2.6 \mathrm{~mm}^{2}$.
\end{abstract}

Index Terms-Averaging, CMOS ISFET, current feedback, current feedback opamp, frequency modulator, ISFET, ISFETs array, log domain, sigma-delta modulator, VCO.

\section{INTRODUCTION}

I ON-SENSITIVE field effect transistors (ISFETs), which were invented by Bergveld [1], are solid-state hydrogen ion $(\mathrm{pH})$ sensors in silicon technology. An ISFET is similar to a field effect transistor (FET) which has a gate, source, drain, and bulk terminals. In contrast to the FET, however, an ISFET's gate comprises an exterior ion-sensitive membrane together with a reference electrode, usually as a silver/silver chloride electrode system. By modifying the chemical composition of the membrane, different chemical sensors such as glucose sensors or DNA sensors [2], [3] are mass producible in commercial CMOS processes at low cost. In the past 10 years, research has focused on integrating ISFETs into a CMOS substrate for experimental [4], portable [1], and implantable [5] applications. One of the drawbacks in these applications is the removal of the polysilicon layer in order to leave the gate oxide of the ISFET in direct contact with the chemical solution. This modification is not compatible with standard CMOS fabrication processes.

Recently, research has explored another direction in what is known as floating-electrode CMOS ISFETs [6], [7]. The CMOS ISFETs are formed by joining a transistor's polysilicon gate with all the metal layers and vias all together. The chip passivation layer, which is a layer of silicon nitride on top of silicon dioxide, becomes the ion-sensitive membrane. Since silicon nitride is amphoteric [1], [6], it can donate or accept positive hy-

Manuscript received September 30, 2009; revised May 24, 2010; accepted May 31, 2010. Date of current version August 25, 2010. This paper was approved by Associate Editor Ken Shepard.

W. P. Chan is with the Department of Electrical and Electronic Engineering, Imperial College London, London SW7 2AZ, U.K. (e-mail: wai.p.chan@imperial.ac.uk).

B. Premanode and C. Touamzou are with the Institute of Biomedical Engineering, Imperial College London, London SW7 2AZ, U.K.

Digital Object Identifier 10.1109/JSSC.2010.2053863 drogen ions. When a silicon nitride is hydrolyzed and charged in an electrolyte, there are equal but opposite charged ions accumulated in the electrolyte just above the surface of the silicon nitride, and those ions are arranged by the electrochemical double layer capacitor [1]. In Nernstian responses, the voltage developed in the double layer capacitor can reach $59 \mathrm{mV}$ in response to a unit change in $\mathrm{pH}$ at room temperature [1]. Silicon nitride has shown a sub-Nernstian sensitivity of $45-56 \mathrm{mV} / \mathrm{pH}$ [8]. By setting up a reference electrode's voltage to define the electrolyte potential, the electrical potential across the double layer capacitor can change the drain current of the ISFET. Thus, there exists a possibility of making chemical sensors in standard CMOS technology, but the potential creates an issue: variations of threshold voltages [6], [7]. Variation occurs as there are undefined trapped charges in the passivation layers, floating metals, and intra-layers dielectric where these charges all contribute to the computation of the threshold voltages. The situation is similar to the trapped charges found in the gate oxide of MOSFETs. In an extreme case, Hammond et al. reported that their p-typed ISFETs had threshold voltages of $-5 \mathrm{~V}$ [9]. This is a big problem in biasing the ISFETs under a $3.3 \mathrm{~V}$ power supply.

There are few other problems in using this solid-state $\mathrm{pH}$ sensor in practice. For instance, ISFETs exhibit short term and long term drifts [10]. Thus, it needs a dedicated algorithm to counteract the drifts. ISFETs also show hysteresis, and recalibration is required for each new experiment. Nevertheless, the possibility of implementing specific functions on a CMOS chip to compensate those ISFETs' performance can produce accurate and precise $\mathrm{pH}$ measurements in micro- or nano-scale.

The aim of this work is to illustrate a microwatt power instrumentation circuit with CMOS ISFETs for handheld instruments. The need for a low power application is very important so as to save batteries' life and to minimize any heat generation due to the electrical power dissipation. Constant temperature minimizes the change in the sensitivity to $\mathrm{pH}$, as ISFETs are sensitive to temperature. Besides, the instrumentation circuit should be able to cope with the uncertainties of the threshold voltages while achieving a satisfactory signal-to-noise ratio for its output. Section II presents the strategy used in the instrumentation. Section III describes the CMOS ISFETs in 1-POLY-6METAL (1P6M) CMOS process and its array implementation. Section IV discusses how the ISFETs array can be adapted into current feedback opamp topology to yield an averaging amplifier. Section V analyzes the microwatt power log domain voltage controlled oscillator which controls the ultimate linearity to the system. Section VI shows the chip measurement results, and Section VII draws a conclusion. 


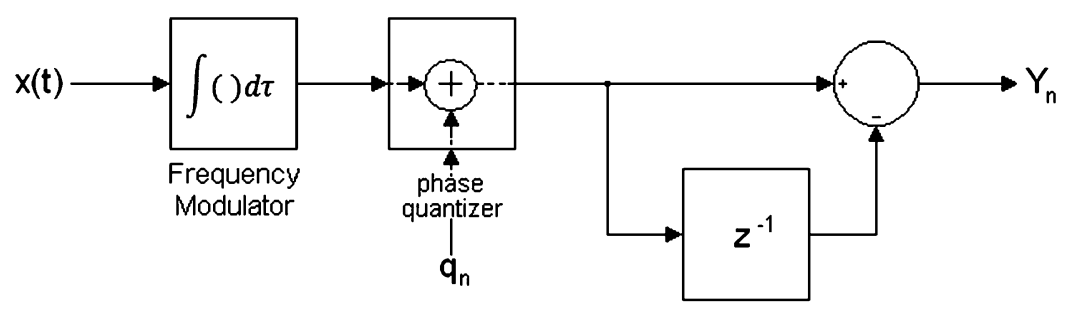

Fig. 1. Open loop first order sigma-delta modulator employing FM.

\section{CHIP ORGANIZATION}

Popular high precision data converters, like SAR converters or integrating analog-to-digital converters, are very good for converting physical analog signals into electronic digital signals. The operation of such converters usually needs a stable reference, either a voltage or current source, to do data comparison and conversion. With negative feedback, the converter can produce an accurate digital representation. These kinds of converters, however, are not suitable for use with CMOS ISFETs readout circuits, as CMOS ISFETs suffer undefined threshold voltages. The difficulty in applying the Nyquist rate data converters involving CMOS ISFETs is determining the right level of the reference value. In an extreme case when the ISFET's threshold voltage is very large, the reference voltage will be too small to turn on the device such that the converter cannot function properly. Therefore, a referenceless converter such as the a first order sigma-delta modulator using frequency modulated intermediate values [11] might be better suited in ISFET-based applications.

The sigma-delta modulator shown in Fig. 1 consists of the frequency modulator, the phase quantizer, and the phase subtracter. The input signal $x(t)$ is frequency modulated according to the following equation:

$$
\varphi(t)=2 \pi \int_{-\infty}^{t}\left(f_{c}+k \cdot x(\tau)\right) d \tau
$$

where $f_{c}$ is the modulator's free running frequency, and $k$ is the gain of the frequency modulator. Thus, the phase content of the modulator embeds signal $x(t)$ which is under integration. After the phase quantization stage, the quantized phase will be subtracted. This restores back signal $x(t)$. On the contrary, the quantization noise $q_{n}$ is differentiated once, so this noise will be first order noise-shaped.

In circuit implementation, a D-flip-flop will be used as the phase quantizer, and the phase subtraction is done by counting the difference of the sum of the previous zero crossing by its current sum. Nevertheless, when the sampling frequency is over twice that of the maximum FM frequency, a D-flip-flop can do the counting, whereas the subtraction can be done using an XOR gate. Hovin [11] has shown that the linear relationship between discrete $Y_{n}$ and continuous $x(t)$ is

$$
Y_{n}=T_{s}\left(f_{c}+k \cdot x(t)\right)+q_{n}-q_{n-1}
$$

assuming that $1 / T_{s} \gg k \cdot x(t)$, and $T_{s}$ is the sampling time, $q_{n}$ is the quantization noise at time $n$, while $q_{n-1}$ is the previous quantization noise at time $n-1$. Equation (2) reveals that the digital output $Y_{n}$ contains the analog input $x\left(t_{n}\right)$ with a gain factor of $T_{s} k$, whereas the quantization noise is first-order differentiated. In the frequency domain, the quantization noise is shaped by $+20 \mathrm{~dB} / \mathrm{decade}$. The whole data conversion does not require any voltage or current reference; it just requires a stable clock. Note that the linearity of this data converter is solely dependent on the gain of the frequency modulator.

The signal to quantization noise ratio (SQNR) of this modulator can be represented as [11]

$$
\mathrm{SQNR}=20 \log \left[\frac{S R_{o}}{2 \sqrt{2}}\right]-10 \log \left[\frac{\pi^{2}}{36} \frac{1}{\mathrm{OSR}^{3}}\right]
$$

where $S R_{o}$ is the full signal output range, which is equal to the amplitude of the analog baseband signal times the gain of the frequency modulator divided by the sampling frequency. OSR is the oversampling ratio between the sampling frequency to two times the maximum baseband frequency. One efficient way to increase the SQNR is by increase the gain of the frequency modulator, subjected to the constraint that the maximum frequency of the intermediate FM signal has to be less than half of the sampling frequency. Otherwise, signal aliasing will occur.

The modulator discussed above will be mapped to the proposed ISFET instrumentation system as shown in Fig. 2, together with its corresponding chip layout plot.

The BIAS GEN shown in Fig. 2 generates bias voltages for the ISFET averaging array, a front-end interface to an array of 64 ISFETs. Note that the ISFETs in Fig. 2 are located on the bottom right region as a rectangular matrix in the layout plot. The ISFET averaging array produces output signals which contain $\mathrm{pH}$ signal, which is equivalent to $x(t)$ as shown in Fig. 1. One of these outputs can be directly monitored via the output port VOUT, or they can be used to bias the on-chip voltage-controlled oscillator (VCO). The output of the VCO can be measured directly through the output port FM, and it also goes directly to the D-flip-flop for phase quantization and subsequent phase subtraction. The signal SD is a digital output representation of the input $\mathrm{pH}$.

\section{CMOS ISFETS ARRAY IMPLEMENTATION}

In this work, an unmodified CMOS process is pursued in making p-typed ISFETs. On the silicon side, the process connects the polysilicon gate layer of a pMOS to all the available six metal layers with vias. These metals are electrically floating in the intra-chip dielectric, as shown in Fig. 3. The topmost chip passivation layer, which is silicon nitride, is used as a $\mathrm{pH}$ sensing layer. Depend on the $\mathrm{pH}$ of a chemical solution, silicon nitride can absorb or donate hydrogen ions, so it can be positively or negatively charged. Due to the electrostatic nature 


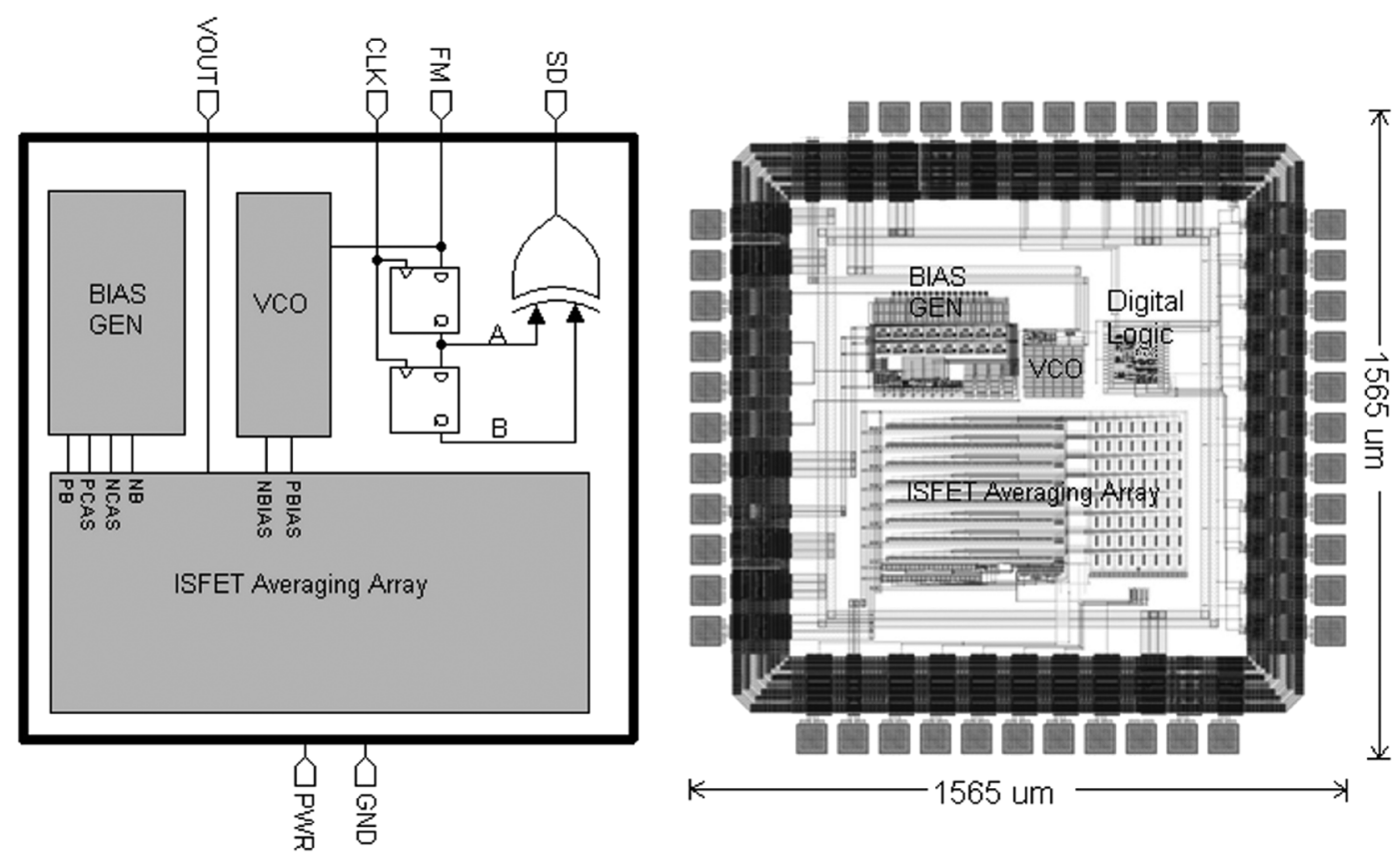

Fig. 2. Functional diagram and chip layout of the integrated CMOS ISFETs instrumentation system.

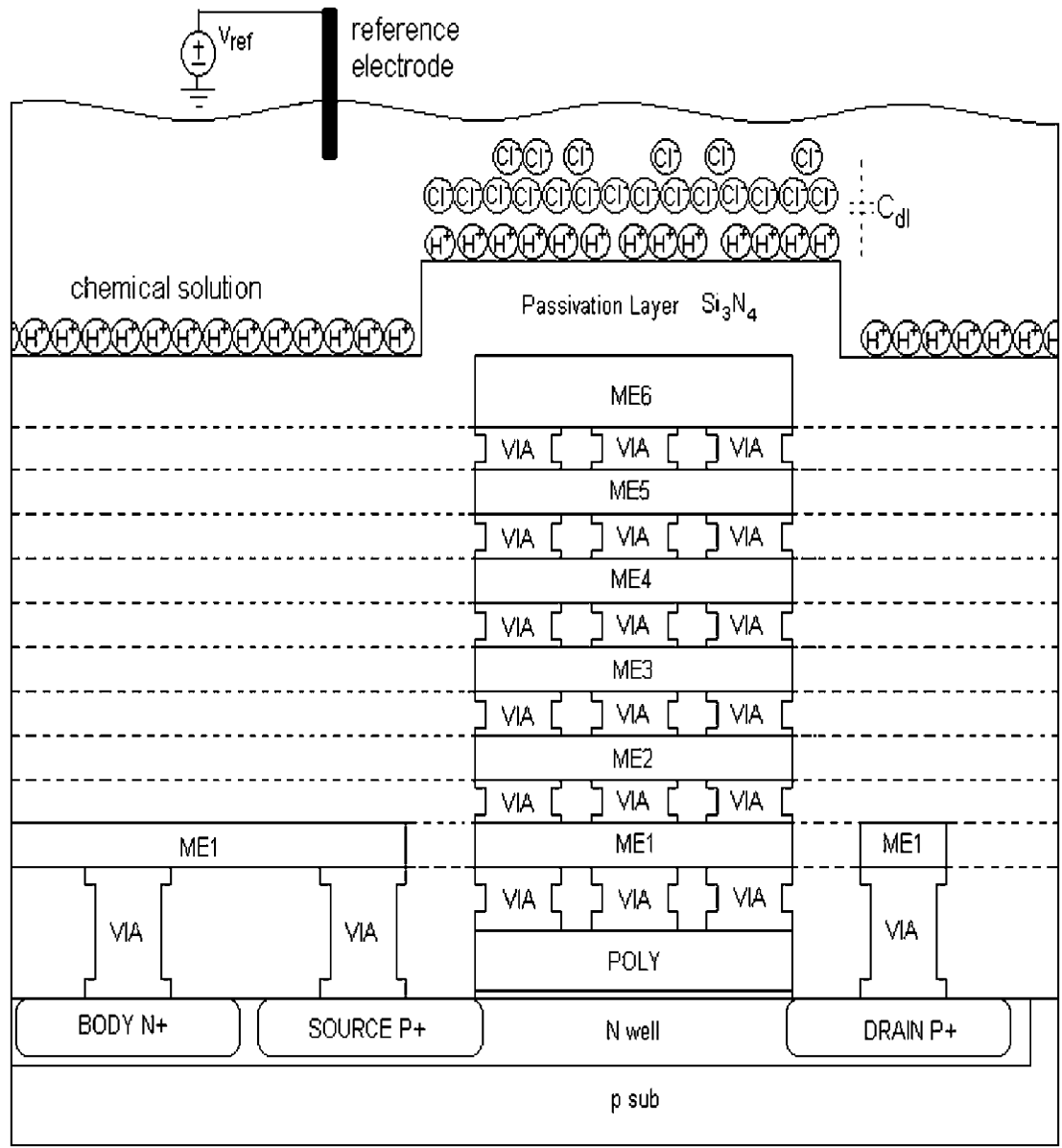

Fig. 3. Cross-section of the CMOS ISFET in 1P6M CMOS process.

of this charged surface, equal but opposite charges are accumulated over the solution in microscopic layers. The charged silicon nitride and the opposite charged layers in the solution form the electrochemical double layer capacitor. The voltage developed in this double layer capacitor is dependent on the amount of charges, so it is $\mathrm{pH}$-dependent. When a reference 


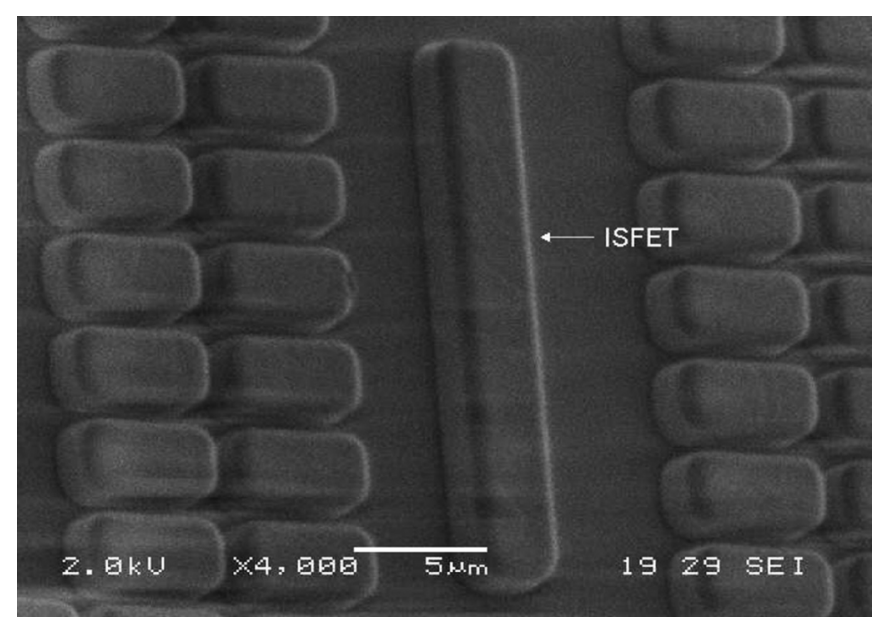

Fig. 4. A SEM image showing the ISFET in its top layer.

electrode is available to define the solution potential, the $\mathrm{pH}$-dependent voltage across the double layer capacitor can modulate the potential at the passivation layer, which in turn modulates the channel current beneath the gate oxide of the transistor. Thus, the drain current of the device can be modulated by $\mathrm{pH}$.

The scanning electron microscope (SEM) image displayed in Fig. 4 helps to illustrate the appearance of the CMOS ISFET over the chip's passivation layer. Note that the rectangular blocks surrounding the ISFET have no effect at all. They are just the same passivation layer covering the top metal fillings.

A reference electrode plays a very important role in forming a complete electrical potential with the ISFET through an electrolyte. In this work, an external $\mathrm{Ag} / \mathrm{AgCl}$ reference electrode was used because it has an exceptional stable potential difference between the silver wire and the solution [12]. Therefore, any solution potential can be set up by applying a voltage bias to the reference electrode. When the solution potential goes down to a certain threshold, the p-typed ISFET will be turned on. As a result, the ISFET outputs current accordingly.

The value of the threshold is equal to the threshold voltage of the ISFET. A simplified model to relate ISFET's threshold voltage can be formulated as [1]

$$
V_{t h}=K+2.3 \alpha U_{T} p H
$$

where the constant $K$, which is $\mathrm{pH}$ independent, cannot be determined accurately because of the trapped charges. The impact of the trapped charges [6] in the passivation layer cannot be found out in advance. In fact, there are variations of the threshold voltages in ISFETs, with extreme values of up to $7 \mathrm{~V}$ in n-typed ISFETs [7].

One possible solution to reduce the impact of the trapped charges is the use of an array. An ISFETs averaging array, which can compute the mean of the samples' threshold voltages, is able to reduce the impact of the variation of the ISFETs' threshold voltages by generating an average one. For instance, this work used 64 samples of p-typed ISFETs in an array configuration. The mean of the 64 samples was assumed to be $-0.7 \mathrm{~V}$, while the standard deviation was assumed to be $0.4 \mathrm{~V}$. Fig. 5(a) shows
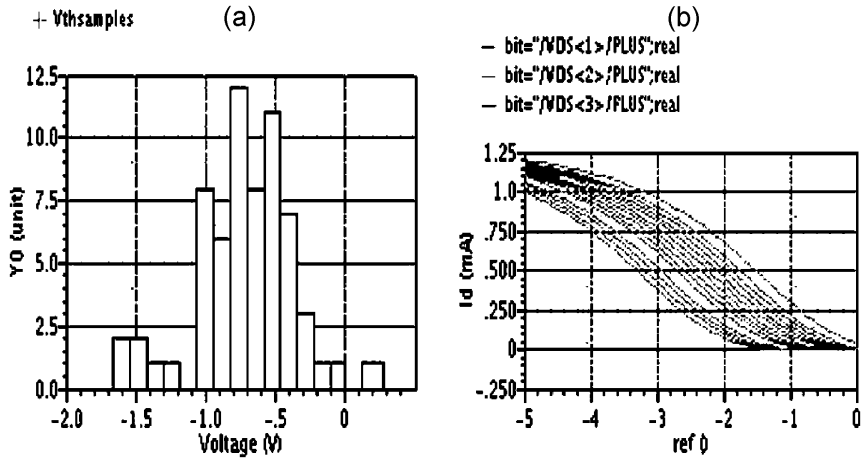

Fig. 5. (a) 64 ISFETs in a normal distribution. (b) Simulated current-voltage transfer characteristics of 64 ISFETs under the same bias conditions and the same aspect ratio.

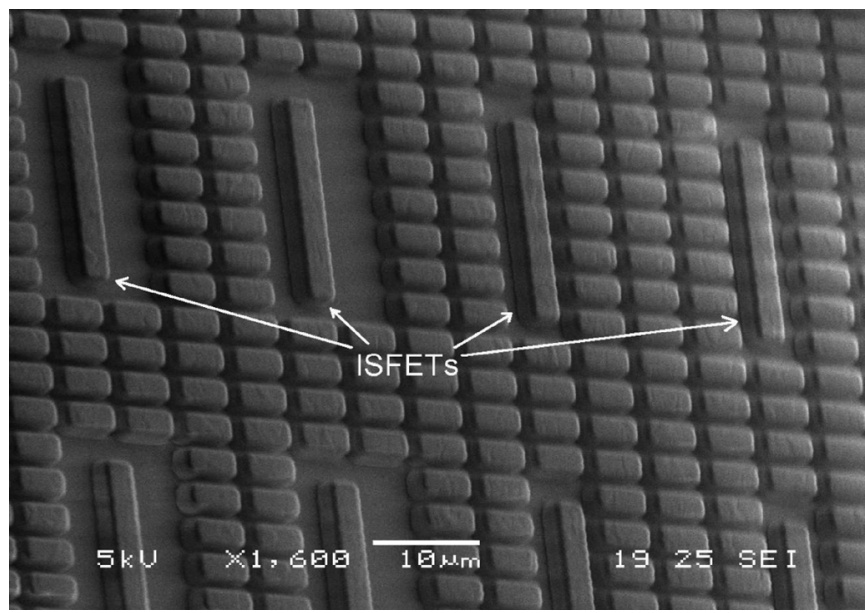

Fig. 6. A SEM image showing the ISFETs arrays.

the threshold voltage distribution of the 64 samples based on the aforementioned statistical properties, while Fig. 5(b) shows the current-voltage transfer characteristics in $0.18 \mu \mathrm{m}$ technology.

Although the statistics are estimated pessimistically, some observations can be made in Fig. 5. First, the 64 samples approximate a normal distribution whose mean is close to $-0.7 \mathrm{~V}$. Second, there are fewer samples whose threshold voltages are in the extreme values. Fig. 5(a) shows the threshold voltages from $-1.7 \mathrm{~V}$ to $0.2 \mathrm{~V}$; the range is about $1.9 \mathrm{~V}$. As a result, the drain current of the samples can be much different, given the same bias condition. In simulation, when the reference electrode is driven by $-2 \mathrm{~V}$, there are about $700 \mu \mathrm{A}$ differences in the samples, which all have the same aspect ratio.

An added advantage of an array implementation is the increase of the surface area to volume ratio over the passivation layer, which can also be visualized by looking at the SEM image in Fig. 6. The longer rectangular blocks represent the passivation layer of the ISFETs array. In this 3-D view, the effective area to volume ratio of the ion-sensitive membrane increases by splitting up a big ISFET into a number of smaller ISFETs. The array is expected to help speed up the chemical equilibrium between the hydrogen ions in the chemical solution and the silicon nitride layer. 


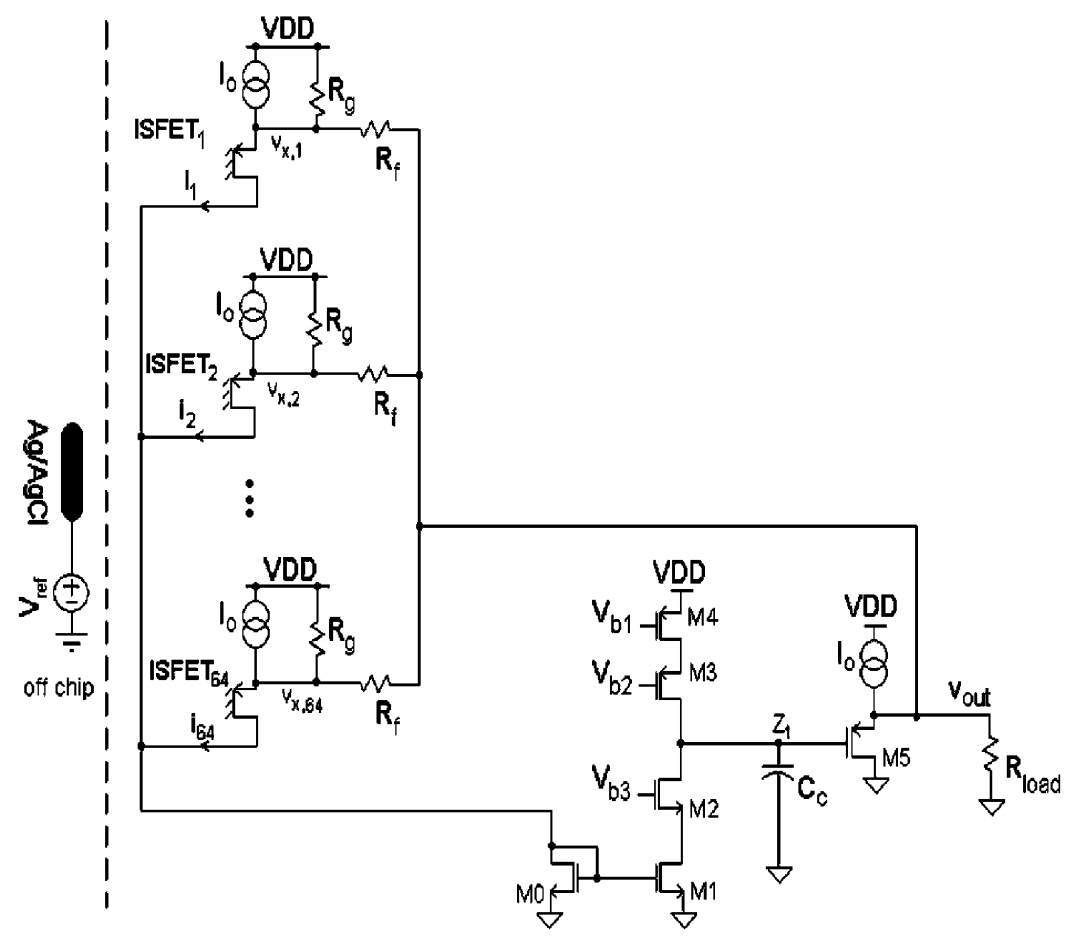

Fig. 7. 64 pH-ISFETs averaging array using a global current feedback.

\section{ISFETS ARRAY INCORPORATED IN CURRENT FEEDBACK TOPOLOGY}

To minimize the impact of the samples' variation of threshold voltages, the ISFETs array described in the previous section has been formatted to compute averages. One of the solutions to bringing up average is employing global negative current feedback as shown in Fig. 7 [13].

Note that $I S F E T_{1}$ to $I S F E T_{64}$ are biased by the constant current source $I_{O}$ to form source follower systems. The choice to use p-type ISFETs is in order to eliminate the back gate effect by shorting the ISFETs' source terminals to their individual substrates. The gate length of the ISFETs is $2 \mu \mathrm{m}$, at which level their channel length modulation parameter is determined to be $0.04 \mathrm{~V}^{-1}$ after empirical extraction of the on-chip devices. Hence, the channel length modulation effect is also negligible. Therefore, the drain current of the ISFETs operating in a weak inversion region can be modeled as [14]

$i_{k}=2 n\left(\frac{w}{L}\right) \mu_{p} C_{o x} U_{T}^{2} \exp \left(\frac{v_{s g, k}+V_{t h, k}}{n U_{T}}\right), \quad k=1 \ldots 64$

where $\mu_{p}$ is the mobility factor, $C_{o x}$ is the gate oxide capacitance, $U_{T}=K T / q$ is the thermal voltage, $n$ is the subthreshold slope factor, and $V_{t h}$ is the threshold voltage of the ISFETs, which is $\mathrm{pH}$ dependent.

Since all the ISFETs are biased by the current source $I_{o}$, and the remote gate voltage is driven by $V_{\text {ref }}$, any change in the $\mathrm{pH}$ of the solution will cause a change in the source terminal voltage $v_{x}$, which can be evaluated by rearranging (5):

$$
v_{x, k}=V_{\text {ref }}-V_{t h, k}+n U_{T}\left(\frac{i_{k}}{2 n \frac{W}{L} \mu_{p} C_{o x} U_{T}^{2}}\right)
$$

By lumping all constants to $C$, the summation of $v_{x}$ can be represented by the summation of the threshold voltages of the ISFETs as in (7):

$$
\begin{aligned}
v_{x, k} & =C_{k}-V_{t h, k} \\
\text { or } \quad \sum_{k}^{64} v_{x, k} & =\sum_{k}^{64} C_{k}-\sum_{k}^{64} V_{t h, k}
\end{aligned}
$$

Summing all the ISFET's drain currents $i_{k}$, where they are under small signal representation, $i_{k}$ can be expressed as

$$
\sum_{k}^{64} i_{k}=\sum_{k}^{64}\left[\frac{v_{x, k}}{R_{g}}+\frac{v_{x, k}-v_{\text {out }}}{R_{f}}\right]
$$

Referring back to Fig. 7, all the ISFETs' drain currents are mirrored and conveyed to the high impedance cascode node $Z_{t}$ via M0 to M4. Subsequently, they are all buffered to the output node $v_{\text {out }}$.

$$
\left(\sum_{k}^{64} i_{k}\right) Z_{t}=v_{\text {out }}
$$

Solving (7), (8), and (9) together and assuming $Z_{t} \gg R_{f}$, $v_{\text {out }}$ can be expressed as

$$
v_{\mathrm{out}}=\left(1+\frac{R_{f}}{R_{g}}\right) \frac{\sum_{k}^{64}\left(C_{k}-V_{t h, k}\right)}{64}
$$

Equation (10) reveals that the output of the amplifier is equal to the closed loop gain of $1+R_{f} / R_{g}$ times the average of the IS- 
(a)

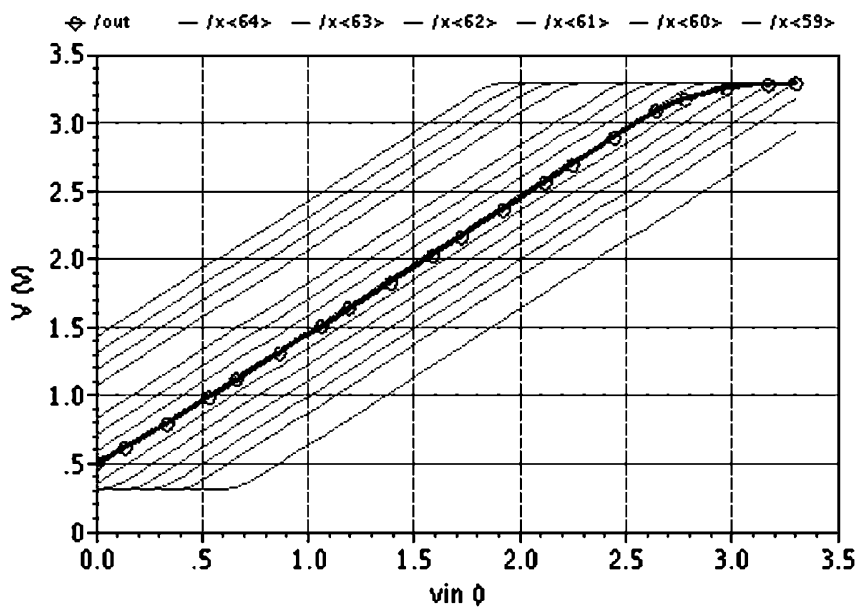

(b)

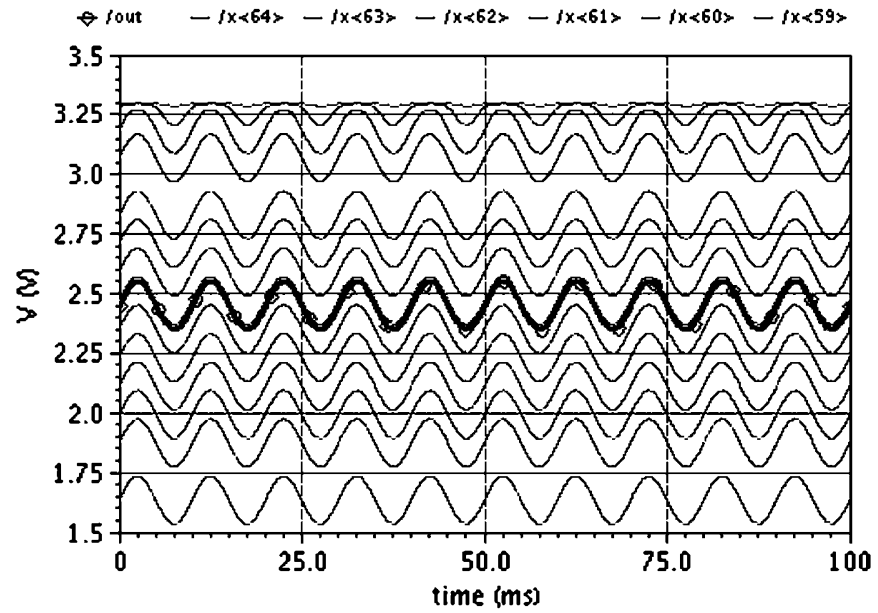

Fig. 8. (a) Transient simulation, and (b) DC simulation of the 64 pH-ISFETs averaging array. The thicker line represents the output of the $64 \mathrm{pH}$-ISFETs averaging array, while the thinner lines represent 64 single-input current feedback amplifiers.

FETs' threshold voltages. Simulation was done using a $0.18 \mu \mathrm{m}$ technology in Cadence Virtuoso Spectre circuit simulator. Fig. 8 illustrates the simulation results of 64 single-input current feedback amplifiers, and a $64 \mathrm{pH}-$ ISFETs averaging array, all configured in a closed loop gain of one. All the ISFETs were sized by the same aspect ratio and subjected to the same current bias. Their threshold voltages followed the normal distribution of a mean $-0.7 \mathrm{~V}$ and a standard deviation of $0.4 \mathrm{~V}$, like what is described in Section III. Fig. 8(a) plots the output voltage versus reference electrode voltage. Even though some of the individual curves saturate toward the power supply voltage, the output of the $64 \mathrm{pH}$-ISFETs averaging array, which emerges as the thicker line situated in the middle of all the curves, extends the linearity range. In fact, the curve is roughly equal to the mean of all the 64 samples. Fig. 8(b) plots the output voltage of the amplifiers in real time with a reference electrode voltage of $2+0.1 \times \sin (2 \pi 100 t) \mathrm{V}$. Some of the curves saturate to the power supply voltage, while some other curves follow the sinusoids with different offsets. However, the output of the 64 pH-ISFETs averaging array, shown as the thicker line, does not appear distorted at all; the extreme samples have no significant effect on the aggregate average.

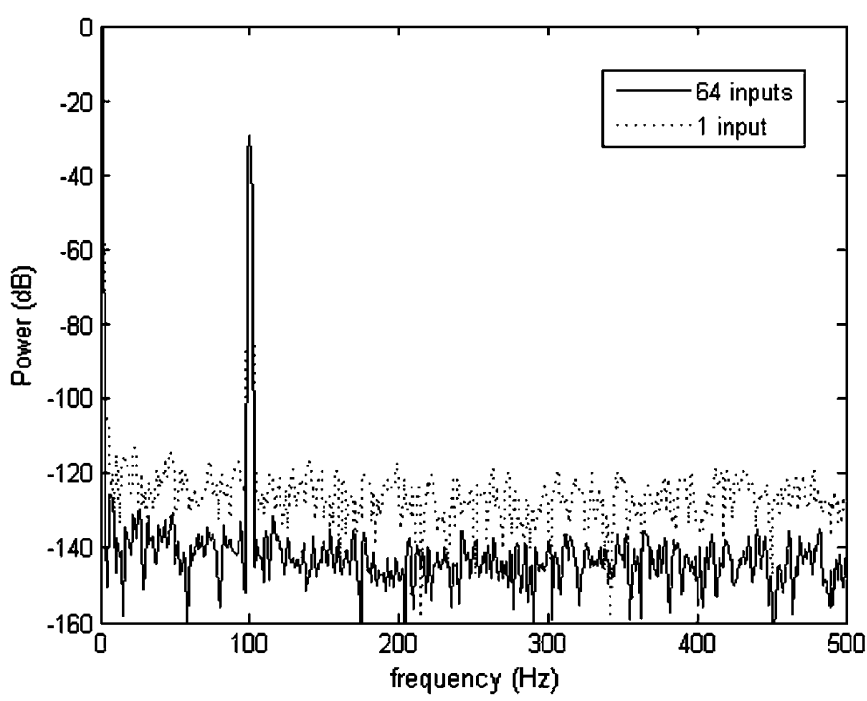

Fig. 9. Simulated frequency spectrum of one ISFET input and 64 ISFETs inputs configured in current feedback opamp topology.

The advantage of using averaging not only reduces the impact of the variations of ISFETs' threshold voltages on the bias point, but also it improves the signal-to-noise ratio to the system [15]. The same schematic set up was done on the transient simulation, but the bias of the reference electrode is $1.4+0.1 \times$ $\sin (2 \pi 100 t) \mathrm{V}$ now. This bias ensures that all the outputs stay in the linear region of the amplifiers. Finally, the simulated data was transferred to Matlab for evaluating their individual power spectral density (psd) so that their spectral characteristics can be compared. In Fig. 9, there are two spikes located at $100 \mathrm{~Hz}$; they are corresponding to the baseband signals. Beside, the noise floor of the one-input current feedback amplifier is about $15 \mathrm{~dB}$ higher than the $64 \mathrm{pH}-\mathrm{ISFETs}$ averaging array. This verifies that the signal-to-noise ratio of the averaging system increases as the sample size of the averaging increases.

Depending on the trapped charges in the passivation layers as well as in the floating metals, it is not easy to implement a single ISFET or even an array of ISFETs on-chip because it is difficult to bias ISFETs with predefined operating point. Therefore, a closed loop gain of one in the ISFETs averaging array was employed in this work to allow headroom for the amplifier to work in the linear region. In order to add amplification to the proposed instrumentation system, a frequency modulator was used; the frequency modulator will be discussed next. Finally, the power consumption will not be a stringent issue as most of the transistors are biased with sub-nano-amps in the weak inversion region.

\section{LOG DOMAIN VOLTAGE-CONTROLLED OSCILLATOR}

The VCO shown in Fig. 1 should ideally be operating as a simple harmonic oscillator, whose transfer function can be represented by the Laplace equation:

$$
\frac{Y(s)}{X(s)}=H(s)=\frac{w_{o}^{2}}{s^{2}+w_{o}^{2}}
$$

where $w_{o}$ is the frequency of oscillation, and $s$ is a complex variable. A simple harmonic oscillator, such as an $L C$ oscillator, can be designed with a high quality factor (high $\mathrm{Q}$ ) such that its 
phase noise is minimized [16], [17]. In this work, an operation of CMOS in the weak inversion region was exploited again to synthesize a microwatt power log domain oscillator. The attractiveness of this VCO is that both its Q value and its frequency of oscillation are tuned independently by two bias currents. It will also be shown that the oscillator is very linear to its controlled current, which can be adjusted by the $\mathrm{pH}$.

Referring back to (11), the Laplace equation representing the simple harmonic oscillator can be transformed to one of its state space equivalent [18]:

$$
\left\{\begin{array}{l}
\dot{X}_{1}=w_{o} X_{2}-w_{o} X_{1} \\
\dot{X}_{2}=w_{o} X_{2}-2 w_{o} X_{1}+w_{o} U \\
Y=X_{1}
\end{array}\right.
$$

where $X_{1}$ and $X_{2}$ are the internal state variables, and $U$ and $Y$ are the input and output, respectively. This set of linear state space equations can be manipulated into a set of exponential state space nodal equations [19], which can reveal the specific circuit topology of the log domain oscillator. The synthesis procedure is as follows.

First, the following exponential state variable mappings are defined:

$$
\left\{\begin{array}{l}
X_{1}=I_{1} \exp \left(\frac{V_{1}}{n U_{T}}\right) \\
X_{2}=I_{2} \exp \left(\frac{V_{2}}{n U_{T}}\right) \\
U=I_{u} \exp \left(\frac{V_{u}}{n U_{T}}\right)
\end{array}\right.
$$

The above mappings are analogs to a MOSFET's drain current in a weak inversion region as listed in (5).

$$
\begin{array}{r}
i_{D}=2_{n}\left(\frac{w}{l}\right) \mu_{n} C_{o x} U_{T}^{2} \exp \left(\frac{v_{g s}-V_{t h}}{n U_{T}}\right) \\
\text { Or in short, } \quad i_{D}=I_{s}\left(\frac{v_{g s}}{n U_{T}}\right)
\end{array}
$$

Thus, (13) is processing in a current mode domain where the state variables, input, and output are all current. Next, the following assignments are chosen:

$$
\left\{\begin{array}{l}
I_{o}=n C w_{o} U_{T} \\
I_{1}=I_{o} \\
I_{2}=I_{s} \\
I_{u}=\frac{I_{s}^{2}}{I_{o}}
\end{array}\right.
$$

Using (13) and (14), the linear state space (12) could be transformed to the following nonlinear KCL equations:

$$
\left\{\begin{array}{l}
C \dot{V}_{1}=I_{s} \exp \left(\frac{V_{2}-V_{1}}{n U_{T}}\right)-I_{o} \\
C \dot{V}_{2}=I_{o}-2 \frac{I_{o}^{2}}{I_{s}} \exp \left(\frac{V_{1}-V_{2}}{n U_{T}}\right)+I_{s} \exp \left(\frac{V_{u}-V_{2}}{n U_{T}}\right) \\
Y=I_{o} \exp \left(\frac{V_{1}}{n U_{T}}\right)
\end{array}\right.
$$

To realize (15) in terms of circuit implementation, Table I employs possible circuit building blocks [18]. Note that the nMOS devices have a triple well option so that their source terminals and the bulk terminals can be tied together individually to minimize the back gate effect.
TABLE I

Circuit Building BLOCKS to REALIZE EquATION (15)

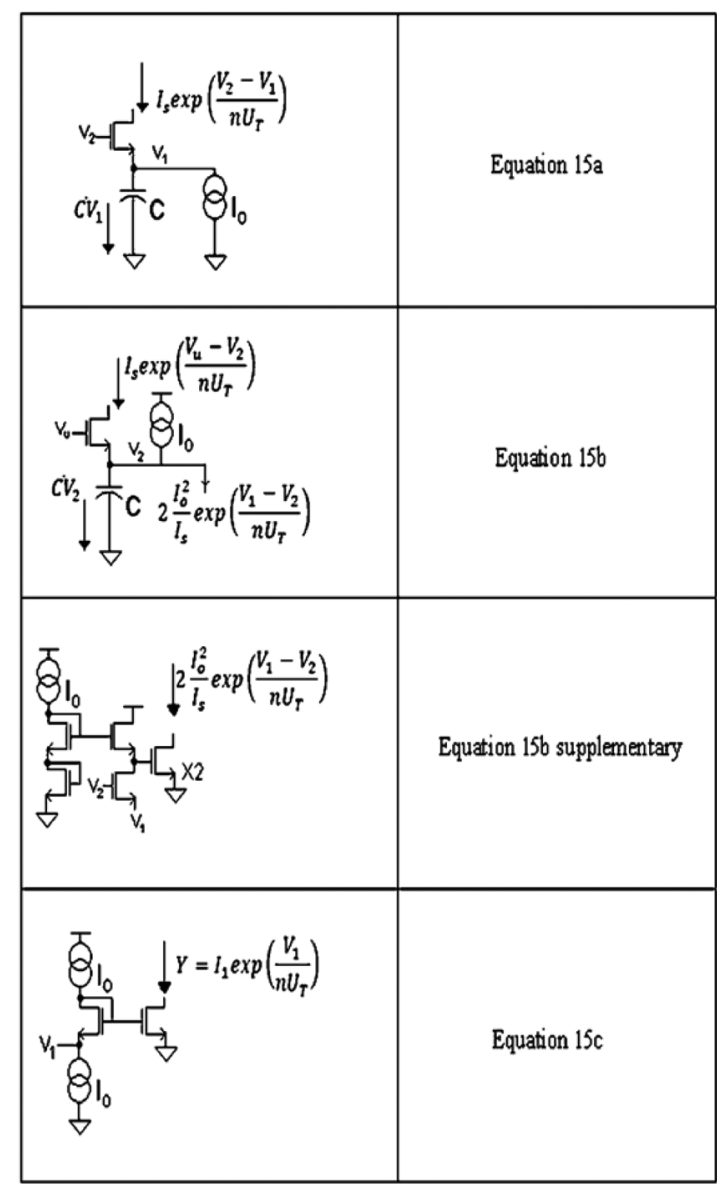

A complete schematic is shown in Fig. 10. It consists of the $64 \mathrm{pH}$-ISFETs averaging array, the voltage-to-current converter, and the log domain oscillator.

The $\mathrm{pH}$ dependent signal $I_{o}$ is set by connecting the ISFETs averaging array with the external resistor $R_{e x t}$ configuring in closed loop gain of one. $I_{o}$ is equal to:

$$
I_{o}=\frac{V_{\text {out }}}{R_{e x t}}
$$

The value of Q and the frequency of oscillation can also be deduced from Fig. 10 [20]. They are:

$$
\left\{\begin{array}{l}
\frac{w_{n}}{Q}=\frac{I_{q}-2 I_{o}}{n C U_{T}} \\
w_{n}=\frac{\sqrt{3 I_{o}^{2}-I_{o} I_{q}}}{n C U_{T}}
\end{array}\right.
$$

If $I_{q}=2 I_{o}$ (which is the ideal case of a simple harmonic oscillator)

$$
\left\{\begin{array}{l}
Q \rightarrow \infty \\
w_{n}=\frac{I_{o}}{n C U_{T}}=\frac{V_{\mathrm{OUT}}}{n R_{\text {ext }} C U_{T}}
\end{array}\right.
$$

Simulations investigated the bias current $I_{q}$ on the $Q$ value of the VCO. The $Q$ value can be inferred by looking at the phase change in the VCO's open loop configuration. The faster the phase changes at its frequency of oscillation, the higher the $Q$ is. Equivalently, the phase noise of the VCO is minimized when 


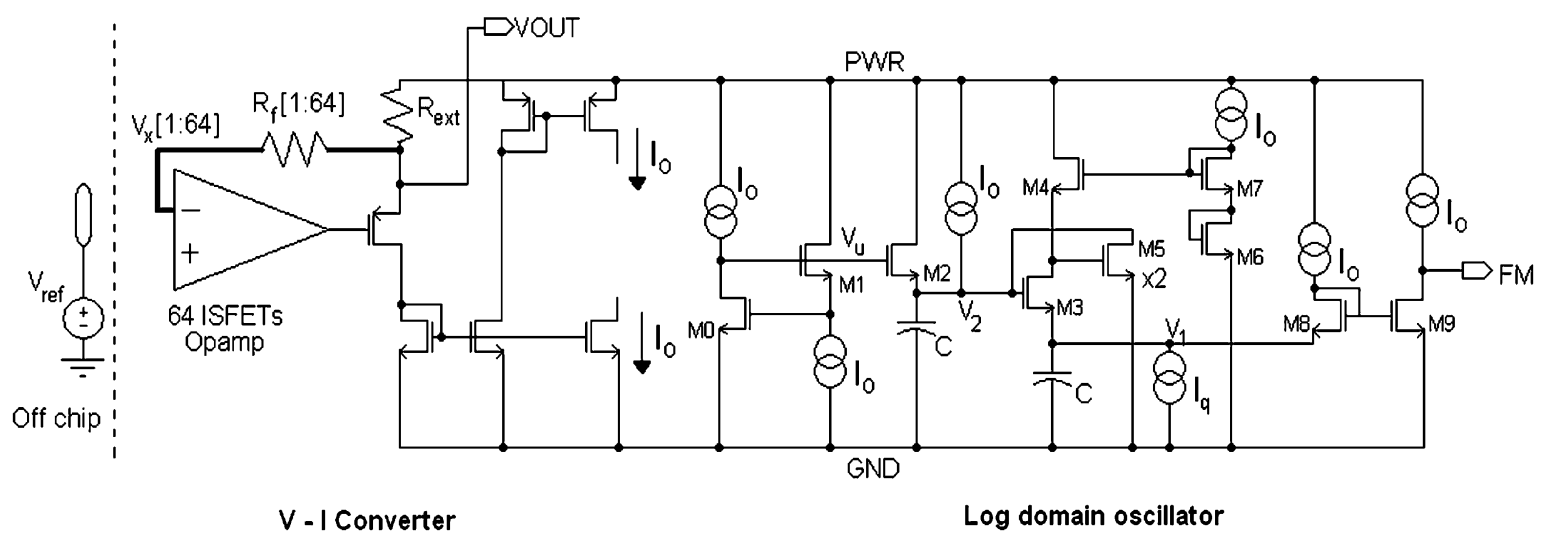

Fig. 10. Schematic diagram showing the log domain vco. The signal $I_{o}$ is determined by the V-I converter consisting of the ISFETs averaging array and the external resistor $R_{\text {ext }}$.

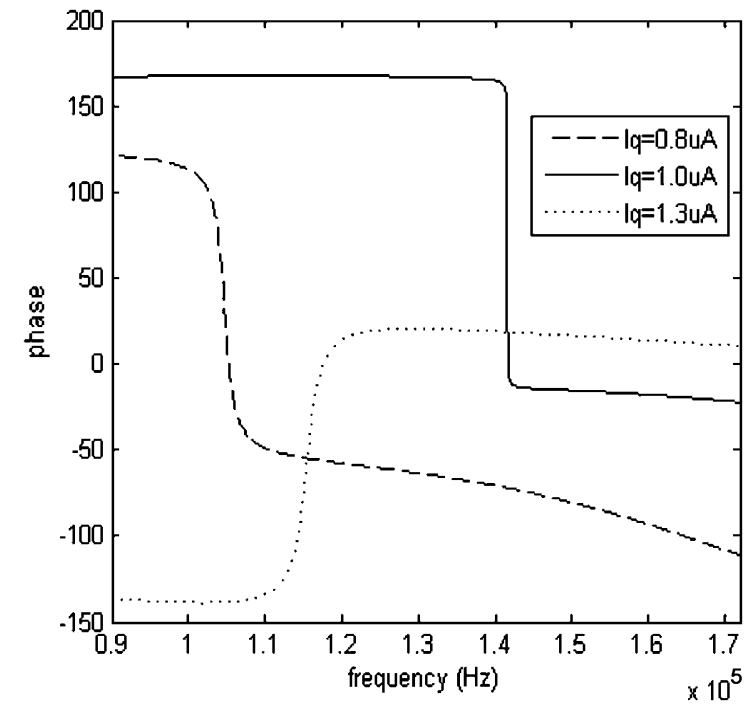

Fig. 11. Simulated open loop VCO's phase spectrum under different $I_{q}$.

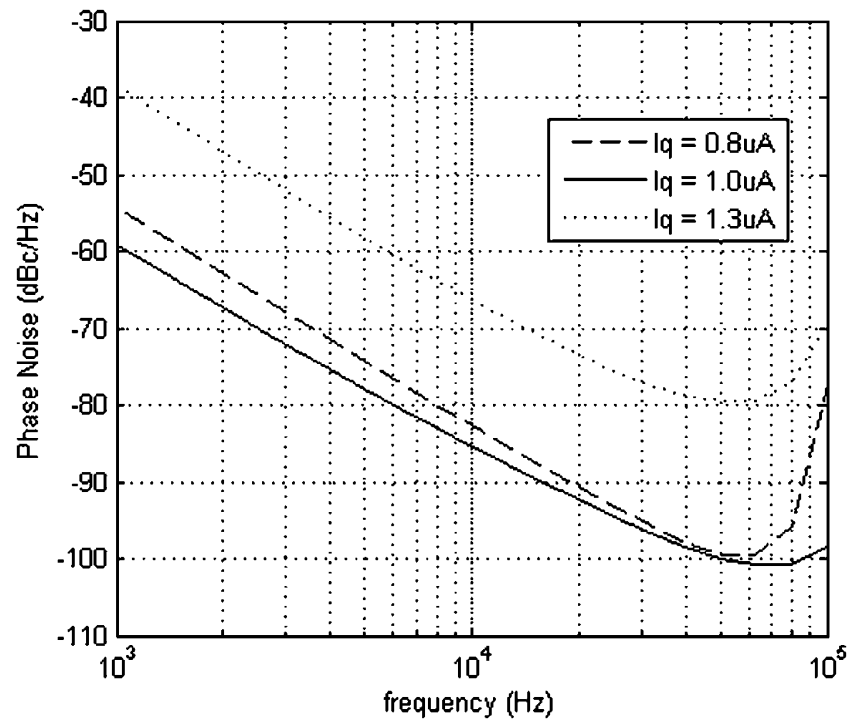

Fig. 12. Simulated VCO's phase noise under different $I_{q}$.

a maximum $\mathrm{Q}$ is attained. These relationships are illustrated in Figs. 11 and 12.

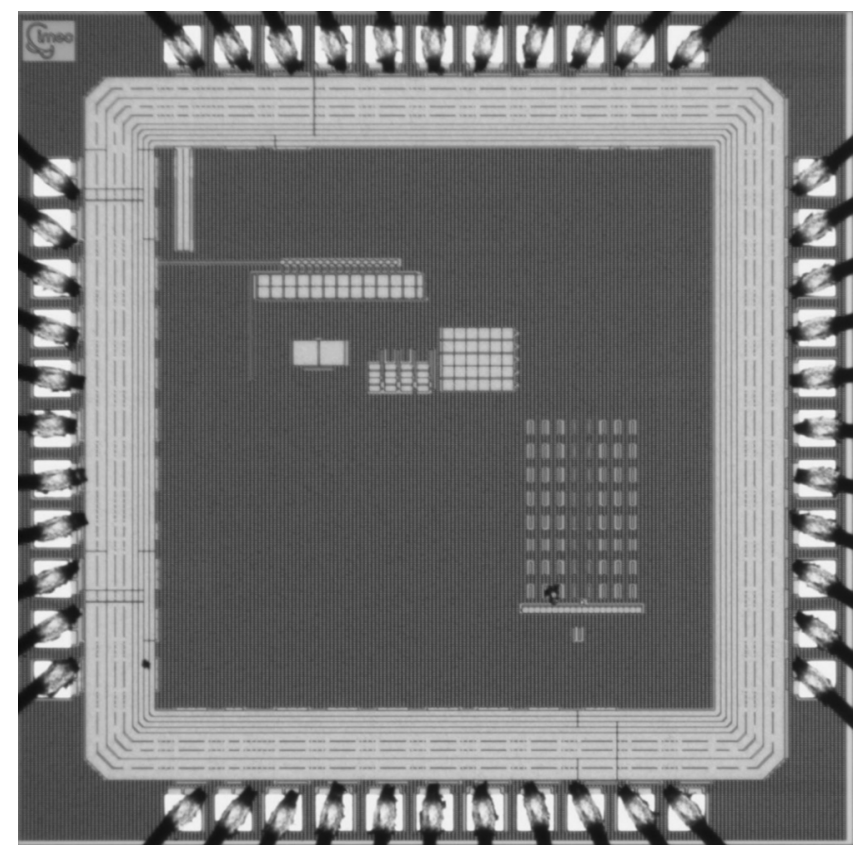

Fig. 13. Chip microphotograph.

Fig. 11 shows the open loop phase response of the VCO. When the value of the bias current $I_{q}=1 \mu \mathrm{A}$, it has the steepest slope at frequency $=150 \mathrm{kHz}$ where the phase is zero degree. All the other $I_{q}$ shows non-optimal Q to the system. Fig. 12 shows the phase noise of the VCO in closed loop, and it reveals that the phase noise is the lowest when $I_{q}=1 \mu \mathrm{A}$.

\section{MEASUREMENT RESUlTS}

Fig. 13 shows the chip microphotograph. The ISFETs array is located in the region in the lower right of the rectangular matrix. The die area is $2.6 \mathrm{~mm}^{2}$.

\section{A. ISFETs Averaging Array Measurements}

First, the electrical properties of the ISFETs averaging array are characterized before investigating the chemical behavior. A DC voltage was swept to the off-chip reference electrode; in this case it was $\mathrm{Ag} / \mathrm{AgCl}$. The reference electrode was used to provide a stable interfacial potential between the electrode and its 


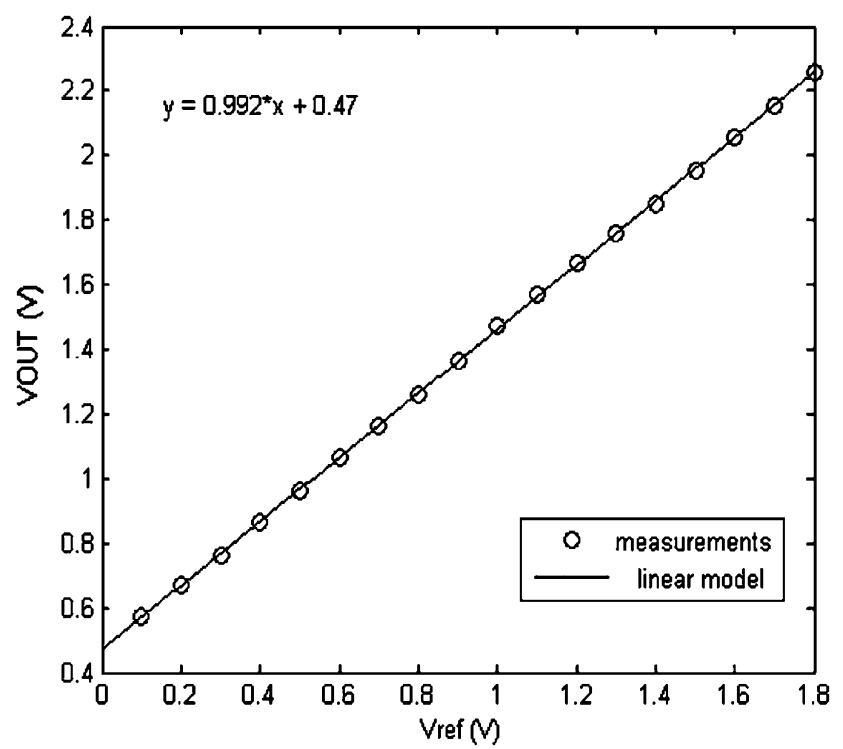

Fig. 14. Measured output voltage of the ISFETs averaging array against the reference electrode's voltage.

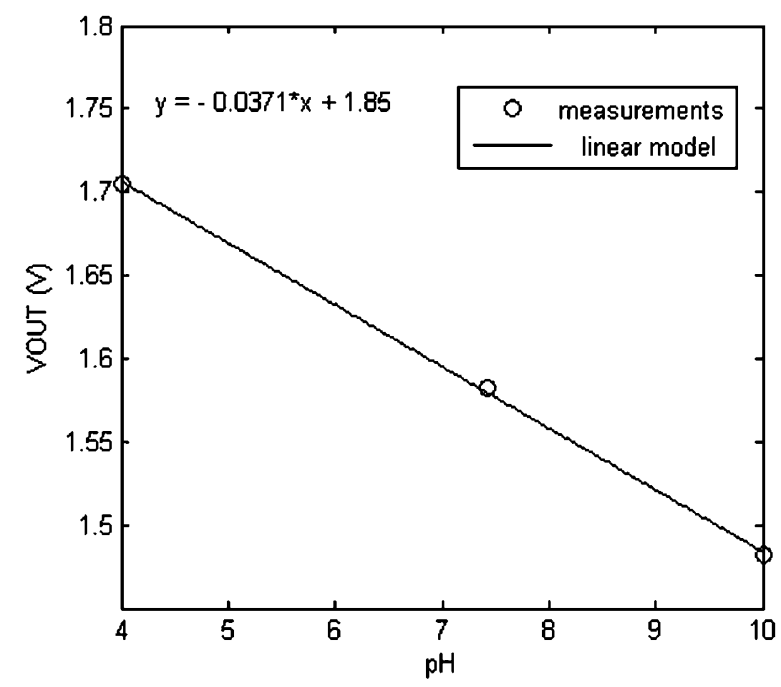

Fig. 15. Measured output characteristics against $\mathrm{pH}$ of the ISFETs averaging array.

surrounding chemical solution. In this way, a complete electrical potential can be set up from the electrode down to the chip's substrate. Next, a Keithley Model 6430 Source Meter was used to sweep the DC voltage on the electrode from $0.1 \mathrm{~V}$ to $1.8 \mathrm{~V}$ in $0.1 \mathrm{~V}$ increments. It was also used to measure the leakage current of the reference electrode. The Source Meter is a good indicator for detecting the leakage current of the ISFETs array. Fig. 14 plots the measured output voltage of the ISFETs averaging array. The result indicates a gain error of less than $1 \%$ in the $1.7 \mathrm{~V}$ output range, which covers more than the full scale range of $\mathrm{pH}$ from 1 to 14 . The measured reference electrode's leakage current for the whole sweep range is less than $10 \mathrm{nA}$ in absolute value.

To characterize the $\mathrm{pH}$ sensitivity of the averaging array, the chip was tested against three chemical buffer solutions: $\mathrm{pH}=4$, 7.4, and 10. The measurements are displayed in Fig. 15. About $37 \mathrm{mV} / \mathrm{pH}$ sensitivity is recorded in this readout; the value is slightly lower than the reported values: $45-56 \mathrm{mV} / \mathrm{pH}$ in silicon

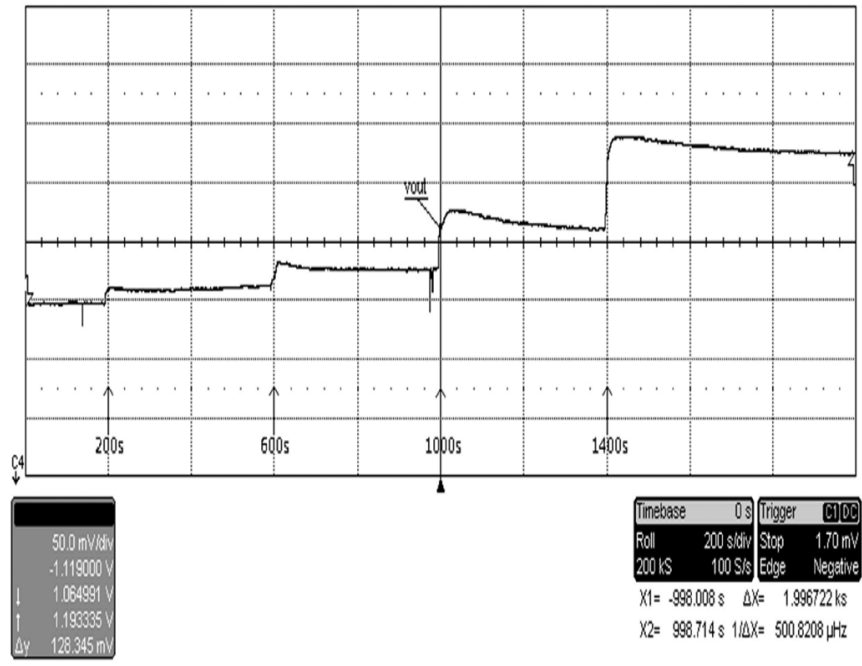

Fig. 16. Measured $\mathrm{pH}$ response of the ISFETs averaging array.

nitride. Finally, real time $\mathrm{pH}$ responses were obtained by exposing the array in N-2-Hydroxyethylpiperazine-N'-2-ethanesulfonic acid (HEPES) at $\mathrm{pH}=7.6$ for an hour. Then, $200 \mu \mathrm{L}$ $0.05 \mathrm{M} \mathrm{H}_{2} \mathrm{SO}_{4}$ was added at $\mathrm{T}=200 \mathrm{~s}, 600 \mathrm{~s}, 1000 \mathrm{~s}$, and $1400 \mathrm{~s}$. The results are displayed in Fig. 16. There are four obvious step changes over the window, which verifies the basic performance of the ISFET averaging array. The strong point of this averaging array is that there is no CMOS post-processing involved. The power consumption of this averaging array is 66 $\mu \mathrm{W}$ approximately.

\section{B. Log Domain VCO}

Recall from Section $\mathrm{V}$ that the frequency sensitivity of the log domain VCO can be controlled by the external resistor $R_{e x t}$. A $10 \mathrm{M} \Omega$ external resistor was used for the test chip, and it generates a bias current $I_{O}$ about $100 \mathrm{nA}$ at $1 \mathrm{~V}$ output. An Agilent Model 4395A Spectrum Analyzer was used to estimate the frequency details of the VCO, and one of the results is shown in Fig. 17. Apart from this frequency spectrum, the VCO gain characteristic is also important because the linearity of the VCO establishes the linearity of the overall instrumentation system. A DC voltage of $0.1 \mathrm{~V}$ incremental sweep across the reference electrode was carried out, and the corresponding output frequency was recorded. These results are displayed in Fig. 18, which shows that the VCO's gain is very linear to the controlled voltage range from $0.5 \mathrm{~V}$ to $1.9 \mathrm{~V}$. The power consumption of this $\mathrm{VCO}$ is below $10 \mu \mathrm{W}$ over the entire input sweep.

\section{Total System}

A $500 \mathrm{kHz}$ sampling clock was chosen to fit the proposed instrumentation system, and the measurements were logged by an oscilloscope from LeCroy. With reference to the signals associated in the instrumentation as shown in Fig. 2, a snapshot of the transient response showing the sampling clock (clk), the frequency modulated signal (FM), and the one bit sigma-delta signal (SD) is displayed in Fig. 19. The same figure shows two internal signals A and B, which were assembled to supplement the functional performance of the sigma-delta modulator. This set of results is based on what the sinusoidal voltage acted on 


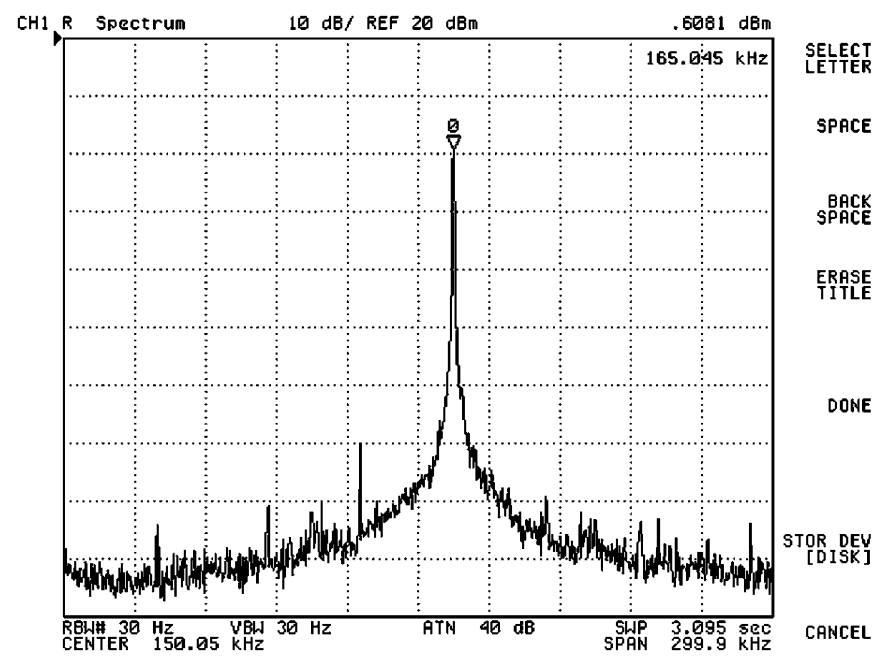

Fig. 17. Measured power spectrum of the log domain VCO when Vref $=$ $1.4 \mathrm{~V}$.

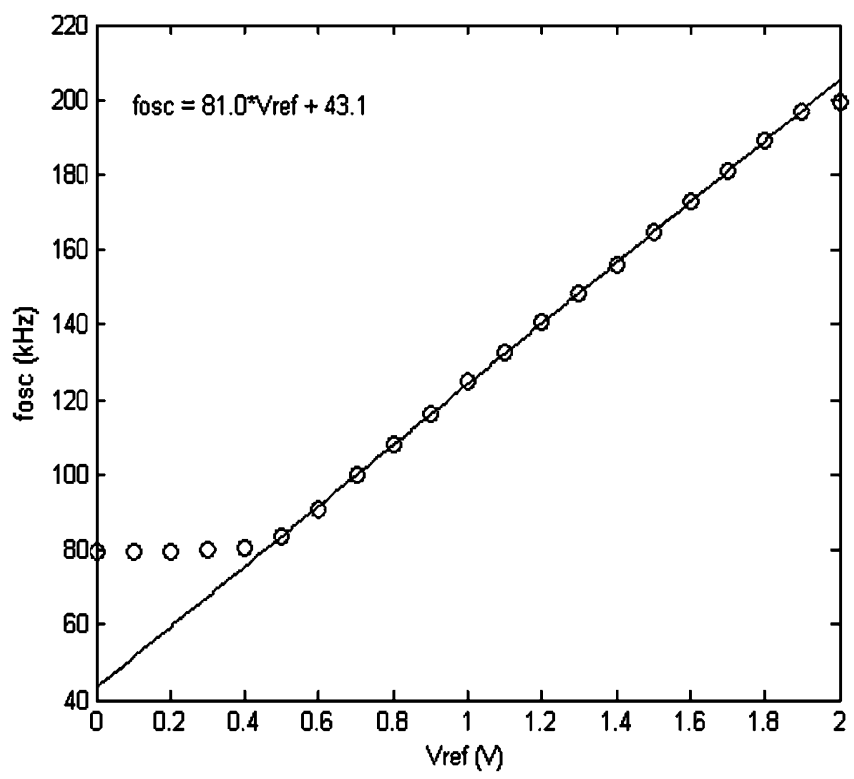

Fig. 18. Measured VCO gain characteristics.

the reference electrode. Note that the signal SD is a direct result of an XOR operation between the internal signals A and B.

The power spectral density (psd) of the signal SD was estimated by Matlab from its $2^{16}$ samples using a Hanning window, and the results are displayed in Fig. 20. Fig. 20 (a) and (b) show the existence of the baseband signals, one at $100 \mathrm{~Hz}$ and the other at $500 \mathrm{~Hz}$. On the other hand, the noise floors displayed in Fig. 20 exhibit a first order noise shaping at frequencies above $10 \mathrm{kHz}$. The SQNR is predicted to be about $45 \mathrm{~dB}$, effectively representing 8 bits of information. The fact that the modulator can produce SQNR of more than $40 \mathrm{~dB}$ with the $500 \mathrm{~Hz}$ baseband signal suggests that the bandwidth of the system can occupy $1 \mathrm{kHz}$. From the above discussions and results, the referenceless sigma-delta modulator achieves 8 bits accuracy in less than $80 \mu \mathrm{W}$.

Spectral analysis might not be a good way to evaluate the performance of the sigma-delta modulator against $\mathrm{pH}$, as the $\mathrm{pH}$ of a chemical solution changes slowly in real time. Since

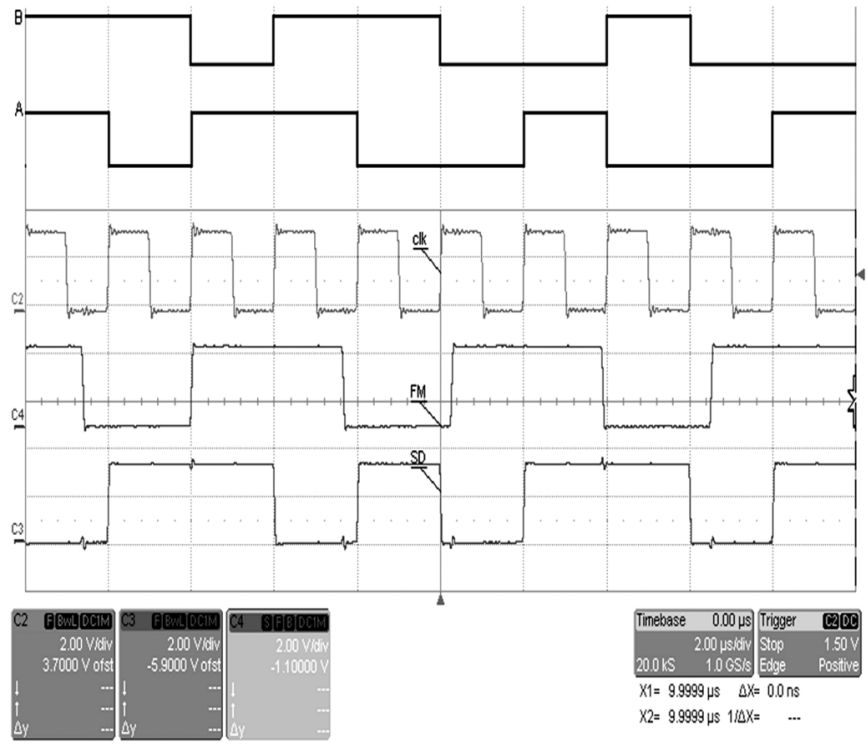

Fig. 19. Transient response showing the main input/outputs of the instrumentation system.

(a)

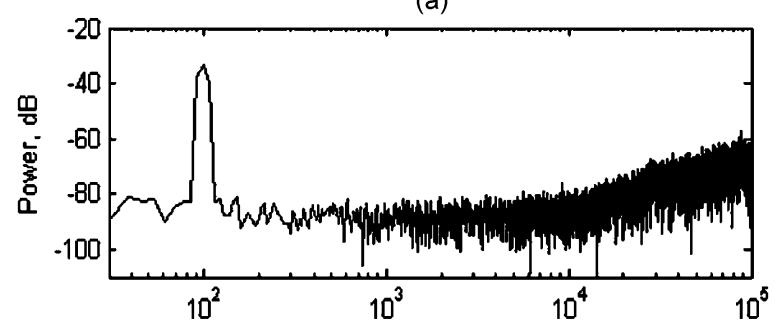

(b)

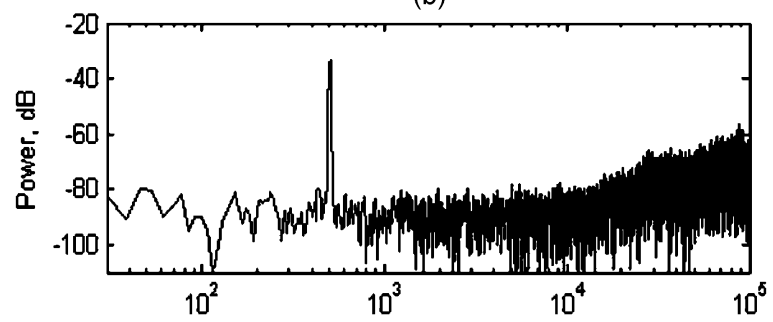

(c)

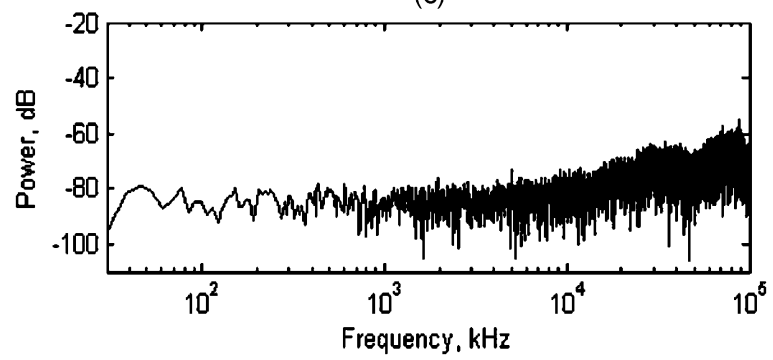

Fig. 20. Measured power spectral density. (a) Input: $1.4+0.12 \times$ $\sin (2 \pi 100 \mathrm{t}) \mathrm{V}$. (b) Input: $1.4+0.12 \times \sin (2 \pi 500 \mathrm{t}) \mathrm{V}$. (c) Input: DC of $1.4 \mathrm{~V}$.

the $\mathrm{pH}$ in a solution is almost constant in case of no chemical disturbance, the modulator will output bitstream data, whose average value in a given period of time is a scaled version of the FM's carrier frequency, as described by (2). Recall that the carrier frequency of the FM signal is dependent on $\mathrm{pH}$, so the easy way to examine the relationship between $\mathrm{pH}$ and the output of the sigma-delta modulator is to evaluate the DC content of 


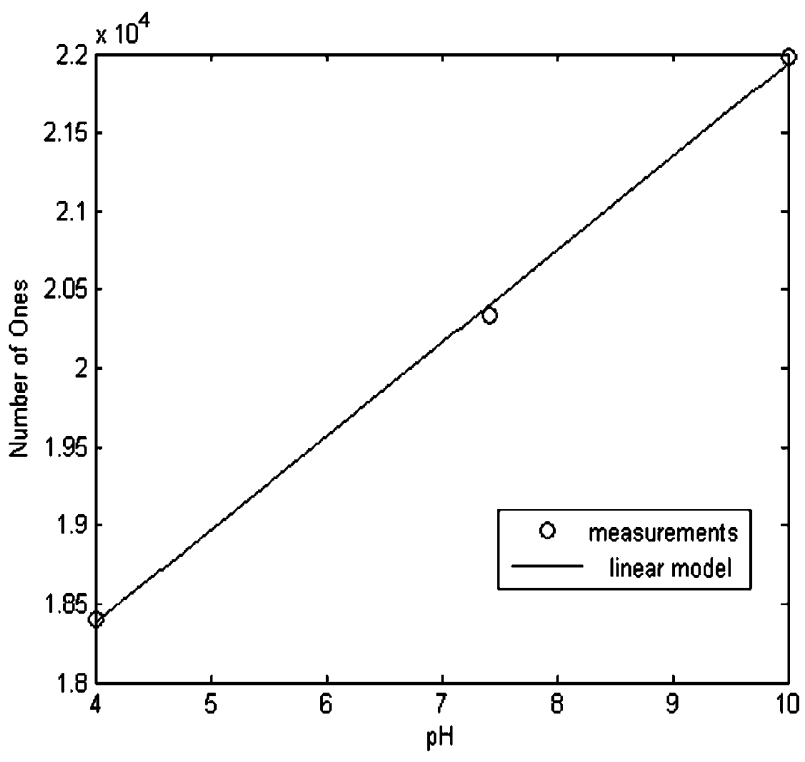

Fig. 21. Measured transfer characteristic between the number of ones in the bitstream output and the $\mathrm{pH}$ input.

TABLE II

PERFORMANCE SUMMARY

\begin{tabular}{ll}
\hline Technology & $0.18 \mu \mathrm{m} \mathrm{1} \mathrm{POLY} \mathrm{6} \mathrm{METAL} \mathrm{CMOS}$ \\
Supply Voltage & $1.8 \mathrm{~V} / 3.3 \mathrm{~V}$ \\
Chip Area & $2.6 \mathrm{~mm}^{2}$ \\
Power Consumption & $76 \mu \mathrm{W}$ (excluding digital core and IO) \\
\hline ISFET Size (W/L) & $20 \mu \mathrm{m} \mathrm{by} 2 \mu \mathrm{m}$ \\
Chemical Sensitivity & $37 \mathrm{mV} / \mathrm{pH}$ (an average of 64 ISFETs) \\
Reference Electrode & Off chip \\
\hline VCO gain & $81 \mathrm{kHz} / \mathrm{V}$ (tunable by an external resistor) \\
Frequency Range & $80 \leftrightarrow 200 \mathrm{kHz}$ \\
\hline Accuracy & $8 \mathrm{bits}$ \\
Bandwidth & $1 \mathrm{kHz}$ \\
Sampling Frequency & $500 \mathrm{kHz}$ \\
\hline \hline
\end{tabular}

the bitstream output. By evaluating the number of ones in the output of the sigma-delta modulator a $100 \mathrm{~ms}$ period, the DC value of the output could be roughly equivalent to the number of ones in the $100 \mathrm{~ms}$ period. However, the counting procedure is post-processed in Matlab.

The experiment consisted of testing the three $\mathrm{pH}$ buffer solutions, $\mathrm{pH}=4,7.42$, and 10 , in the chip again and counting the number of ones in the output of the sigma-delta modulator. The count began when the ISFETs' responses reach a steady state. The result is displayed in Fig. 21. The circle represents the measured number of ones, while the straight line represents the linear regression model. The data shows a good match between the digital output and the chemical input with less than $0.5 \%$ error in predicting the linear model.

Finally, a performance summary of the instrumentation system is listed in Table II.

\section{CONCLUSION}

An integrated ISFETs instrumentation system in a standard CMOS process has been presented. The instrumentation regulates the undefined ISFETs' threshold voltages by using the averaging array, which is based on a global negative current feedback technique. Moreover, signal amplification is achieved in a frequency domain by employing the microwatt power VCO to transform a baseband signal into a frequency modulated signal. The wide voltage tuning range together with the independent $\mathrm{Q}$ control makes the log domain VCO a good frequency modulator for use in the referenceless sigma-delta modulator. Finally, the sigma-delta modulator is able to perform a linear relationship between the $\mathrm{pH}$ input and the digital output, and it provides up to 8 bits accuracy under $80 \mu \mathrm{W}$ power consumption.

\section{ACKNOWLEDGMENT}

The authors would like to thank Maria Khaleeq from the High Energy Physics Group, Imperial College, who wirebonded the chip onto the printed circuit board, and Mahmoud Ardakani from the Department of Materials, Imperial College, for taking the SEM images.

\section{REFERENCES}

[1] P. Bergveld, "Thirty years of ISFETOLOGY. What happened in the past 30 years and what may happen in the next 30 years," Sensors and Actuators B, vol. 88, pp. 1-20, 2003.

[2] Y. Hanazato, M. Nakako, S. Shiono, and M. Maeda, "Integrated multibiosensors based on an ion-sensitive field-effect transistor using photolithographic techniques," IEEE Trans. Electron. Devices, vol. 36, pp. 1303-1310, 1989.

[3] S. Purushothaman, C. Toumazou, and C. Ou, "Protons and single nucleotide polymorphism detection: A simple use for the ion sensitive field effect transistor," Sensors and Actuators B, vol. 114, pp. 964-968, 2006.

[4] E. Lauwers et al., "A CMOS multiparameter biochemical microsensor with temperature control and signal interfacing," IEEE J. Solid-State Circuits, vol. 36, pp. 2030-2038, 2001.

[5] S. J. Schepel et al., "In vivo experiments with a pH-ISFET electrode," Med. Biol. Eng. Comput., vol. 22, pp. 6-11, 1984.

[6] P. A. Hammond and D. R. S. Cumming, "Performance and system-onchip integration of an unmodified CMOS ISFET," Sensors and Actuators B, vol. 111-112, pp. 254-258, 2005.

[7] J. Bausells, J. Carrabina, A. Errachid, and A. Merlos, "Ions-sensitive field effect transistors fabricated in a commercial CMOS technology," Sensors and Actuators B, vol. 57, pp. 56-62, 1999.

[8] H. Abe, M. Esashi, and T. Matsuo, "ISFET's using inorganic gate thin films," IEEE Trans. Electron. Devices, vol. 26, pp. 1939-1944, 1979.

[9] P. A. Hammond, D. Ali, and D. R. S. Cumming, "Design of a singlechip $\mathrm{pH}$ sensor using a conventional 0.6- $\mu \mathrm{m}$ CMOS process," IEEE Sensors J., vol. 4, pp. 706-712, 2004.

[10] S. Jamasb, S. D. Collins, and R. L. Smith, "A physical model for threshold voltage instability in $\mathrm{Si}_{3} \mathrm{~N}_{4}$-gate $\mathrm{H}^{+}$-sensitive FET's $(\mathrm{pH}$ ISFET's)," IEEE Trans. Electron. Devices, vol. 45, pp. 1239-1245, 1998.

[11] M. Hovin, A. Olsen, T. S. Lande, and C. Toumazou, "Delta-sigma modulators using frequency modulated intermediate values," IEEE $J$. Solid-State Circuits, vol. 32, pp. 13-22, 1997.

[12] R. Cattrall, Chemical Sensors. : Oxford Uni. Press, 1997.

[13] W. P. Chan, B. Premanode, and C. Toumazou, "64 pH-ISFET averaging array employing global negative current feedback," IET Electron Lett., vol. 45, pp. 536-537, 2009.

[14] Y. Tsividis, Operation and Modeling of the MOS Transistor. Oxford, U.K.: Oxford University Press, 1999.

[15] D. John and K. Martin, Analog Integrated Circuit Design. New York: Wiley, 1997.

[16] B. Razavi, "A study of phase noise in CMOS oscillators," IEEE J. Solid-State Circuits, vol. 31, pp. 331-343, 1996.

[17] T. Lee and A. Hajimiri, "Oscillator phase noise: A tutorial," IEEE J. Solid-State Circuits, vol. 35, pp. 326-336, 2000.

[18] J. Ngarmnil, A. Thanachayanont, and C. Toumazou, "On the design of micropower active resonators and oscillators using log domain processing," in Proc. 3rd Int. Conf. Electronics, Circuits, and Systems, 1996, vol. 1, pp. 207-210.

[19] D. Frey, "Exponential state space filters: A generic current mode design strategy," IEEE Trans. Circuits Syst. I, Fundamental Theory and Applications, vol. 43, pp. 34-42, 1996

[20] W. P. Chan, "Robust low power CMOS methodologies for ISFETs instrumentation," Ph.D. dissertation, Imperial College London, London, U.K., 2010 


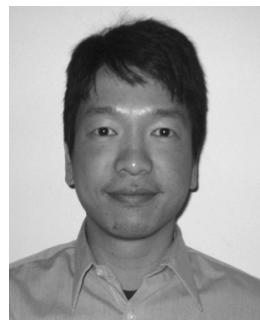

Wai Pan Chan received the B.Sc. degree in electrical engineering (summa cum laude) from North Carolina State University, Raleigh, in 2004, and the Ph.D. degree in electrical engineering from the Imperial College London, London, U.K., in 2010.

$\mathrm{He}$ is currently with the Institute of Biomedical Engineering at Imperial College London, where he is working as a research associate in ISFET Sensor Technology. His research interests include CMOSbased chemical sensing technology for DNA detections, low power circuit designs, and current mode signal processing designs.

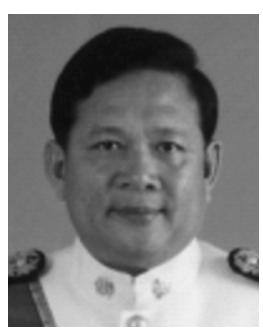

Bhusana Premanode received the B.Sc. degree from Kasetsart University, Thailand, the M.B.A. in a joint management course by the Wharton School of the University of Pennsylvania and Kellogg School of Management, Northwestern University, Evanston, IL, the Ph.D. from Imperial College London, U.K., and the D.B.A. from Somerset University, U.K.

He joined Imperial College, London as a Visiting Professor in the Department of Bioengineering in 2000 and moved to the Institute of Biomedical Engineering in late 2006. He is doing research in biosensors, especially analog design and testing methodologies for in vitro peritoneal dialysis machine. He was a founder and CEO of one of the largest mobile phone public companies in Thailand, where he and Prof. C. Toumazou designed dual-mode GSM phone, narrowband GSM repeaters and many others. $\mathrm{He}$ is the author or coauthor of over 18 papers in biomedical science and holds two international patents. He is currently pursuing his third Ph.D. degree, in quantitative finance.

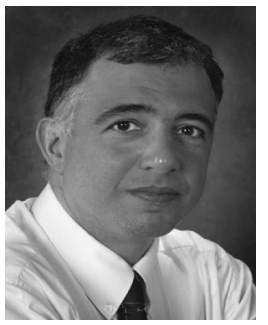

Christofer Toumazou (M'87-SM'99-F'01) received the Ph.D. degree from Oxford Brookes University in collaboration with the University of Manchester Institute of Science and Technology, U.K., in 1986.

$\mathrm{He}$ is a Professor of Circuit Design with the Department of Electrical and Electronic Engineering, Imperial College London, U.K., where he is the Founder and Director of the Institute of Biomedical Engineering. His research interests include high-frequency analog integrated circuit design in bipolar, CMOS, and SiGe technology for RF electronics and low-power electronics for biomedical applications. He has authored or coauthored some 300 publications in the field of analog electronics. He is also the Founder and Director of Toumaz Technology Ltd., Abingdon, U.K. He is currently an Honorary Editor and Chairman of the Institution of Electrical Engineers (IEE) Electronics Letters.

Prof. Toumazou is a past Chairman for the Analog Signal Processing Committee and the past Vice President of Technical Activities for the IEEE Circuits and Systems (CAS) Society. He is a member of many professional committees. He was the Editor-in-Chief of the IEEE TRANSACTIONS ON CIRCUITS AND Systems II: ANALOG AND Digital Signal ProceSSING. He is also a recipient of the 1992 IEEE CAS Outstanding Young Author Award for his work on high speed GaAs operational-amplifier design. He is a co-winner of the IEE (U.K.) 1991 Rayleigh Best Book Award for Analog IC Design: The Current-Mode Approach. 